\title{
Future trends: Adding Computational Intelligence, Knowledge and Creativity to Interactive Exhibits and Visualisation Systems
}

\author{
Steve DiPaola \\ Simon Fraser University \\ 8888 University Dr Burnaby BC \\ Canada V5A 1 S6 \\ sdipaola@sfu.ca
}

\section{KEYNOTE ABSTRACT}

Computational advances are heralding in new ways of interactively expressing a body of work or complicated narrative to a galley, art or science museum audience. These more socially-based interactive visualization and experiential systems, while computer based, can bend interactive technologies more to the human experience by incorporating human knowledge, expressive and creativity models into the system. For example, high end game based talking faces that express passion via a narrative about a carved native mask or science exhibition: Artificial intelligent 3D virtual whales that allow interaction with aquarium visitors to experimentally understand, in the wild, pod behaviours and scenarios: teaching evolution through evolving interactive art exhibitions and other new techniques are beginning to change the possibilities for engaging, communicating, teaching and interacting with gallery and museum visitors. 